\title{
Scanning apertureless microscopy below the diffraction limit: Comparisons between theory and experiment
}

\author{
C. J. Hill, P. M. Bridger, G. S. Picus, and T. C. McGilla) \\ Thomas J. Watson, Sr. Laboratory of Applied Physics, 128-95, California Institute of Technology, \\ Pasadena, California 91125
}

(Received 23 April 1999; accepted for publication 27 October 1999)

\begin{abstract}
The exact nature of the signal in scanning apertureless microscopy techniques is the subject of much debate. We have sought to resolve this controversy by carrying out simulations and experiments on the same structures. Simulations of a model of tip-sample coupling are shown to exhibit features that are in agreement with experimental observations at dimensions below the diffraction limit. The simulation of the optical imaging process is carried out using atomic force microscope data as a topographical template and a tip-sample dipole coupling model as the source of optical signal. The simulations show a number of key fingerprints including a dependence on the polarization of the external laser source, the size of the tip, and index of refraction of the sample being imaged. The experimental results are found to be in agreement with many of the features of the simulations. We conclude that the results of the dipole coupling theory agree qualitatively with experimental data and that apertureless microscopy measures optical properties, not just topography. (C) 1999 American Institute of Physics. [S0003-6951(99)02451-1]
\end{abstract}

The attainment of the goal of imaging structures on the near-atomic scale could revolutionize chemistry, biology, and condensed matter physics. To this end, many imaging techniques have been developed which claim to measure optical properties at resolutions well below the classical diffraction limit for the illuminating wavelength. One of the most promising of these is the scanning apertureless microscope (SAM) technique pioneered by Wickramasinghe. ${ }^{1,2}$ Yet, as is often true with the proximal probe techniques, there have been a number of questions about what exactly is measured. Some of the controversy centers around whether or not one can separate information that is topographical in nature from that which is optical in any near-field imaging technique. ${ }^{3}$ In this letter, we present both simulations and experiment which show that the technique measures optical properties of the sample not accessible by a straightforward atomic force microscope (AFM) measurement.

The experimental setup upon which the analysis is based consists of a Digital Instruments Bioscope AFM operating in tapping mode, with the AFM tip illuminated by an external HeNe laser source through the camera port of a Nikon Diaphot 200 which forms the base of the bioscope. The signal scattered off of the tip is collected and then filtered by a lock-in amplifier with the tip oscillation frequency as a reference. This technique allows the simultaneous measurement of sample topography with the AFM as well as information that is ostensibly optical in nature with the SAM. A simplified schematic of the experimental setup is shown in Fig. 1. An in-depth discussion of the experimental technique can be found in the paper by Bridger. ${ }^{4}$

The optical image is the direct measurement of the intensity of the radiation scattered by the AFM tip at the oscillation frequency. In our simulations we model the AFM tip as a radiating dipole, where the expression for the far-

${ }^{a)}$ Electronic mail: tcm@ssdp.caltech.edu field intensity pattern produced by such a dipole is

$$
I_{t}=\frac{c \epsilon_{0} k^{4}}{\left(4 \pi \epsilon_{0}\right)^{2} d^{2}}\left\langle\left|\mathbf{p}_{t}\right|^{2}\right\rangle \sin ^{2} \phi
$$

where $I_{t}$ is the intensity, $\mathbf{p}_{t}$ is the dipole moment of the tip, $d$ is the distance to the detector, $k$ is the wave number of the radiation, $c$ is the speed of light, $\epsilon_{0}$ is the permittivity of free space, and $\phi$ is the angle between the vector from the tip to the detector and the dipole moment of the tip. ${ }^{5}$ The term in the above equation that contains optical information about the sample is $\mathbf{p}_{t}$, the dipole moment of the tip, which is given by

$$
\mathbf{p}_{t}=4 \pi \epsilon_{0} \alpha_{t}\left(\mathbf{E}_{\text {laser }}+\mathbf{E}_{s}\right),
$$

where $\alpha_{t}$ is the polarizability of the tip. The total field incident on the tip is the sum of the laser field $\mathbf{E}_{\text {laser }}$ and the sample's field $\mathbf{E}_{s}$, therefore, it is necessary to calculate the very near field of the sample in order to determine the farfield radiation pattern produced by the tip.

For simplicity, we model the features being imaged as dielectric nanospheres with an induced dipole moment which, to first order, is $\mathbf{p}_{s}=4 \pi \epsilon_{0} \alpha_{s} \mathbf{E}_{\text {laser }}$, where all of the symbols are the same as before except that they refer to the

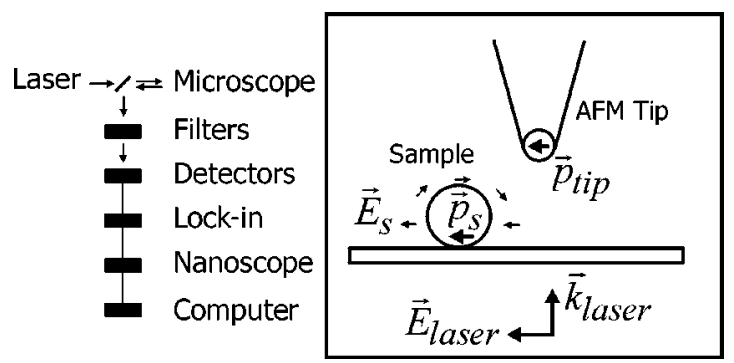

FIG. 1. Schematic of experimental setup. The inset gives a simplified view of the interaction geometry. 


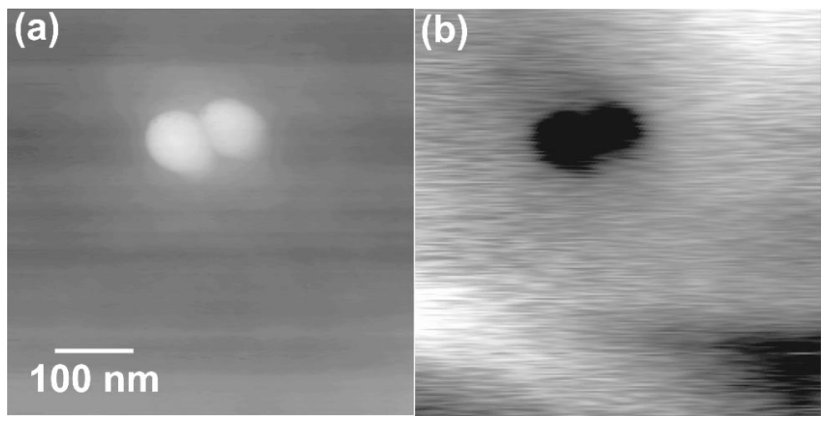

FIG. 2. (a) AFM and (b) SAM (random source polarization) images of two adjacent $50 \mathrm{~nm}$ polystyrene nanospheres.

sample instead of the tip. The value of the polarizability $\alpha_{s}$ is dependent on the structure of the sample and the wavelength of the external laser, and for spheres is expressed as

$$
\alpha_{s}=\frac{\epsilon_{s} / \epsilon_{0}-1}{\epsilon_{s} / \epsilon_{0}+2} R_{s}^{3}
$$

where $R_{s}$ is the radius and $\epsilon_{s}$ is the dielectric constant of the sphere. ${ }^{5}$ The dependence on the index of refraction is contained in $\epsilon_{s}$, since $n=\sqrt{\epsilon_{s}}$. The full expression for the near
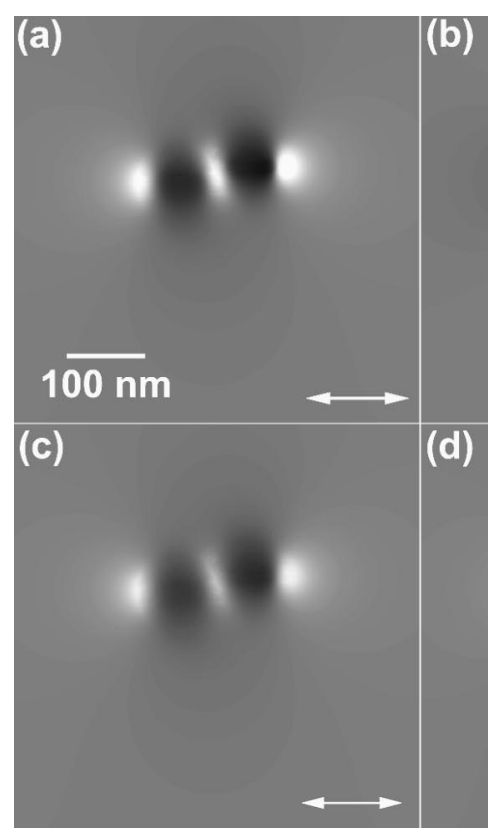

(d)
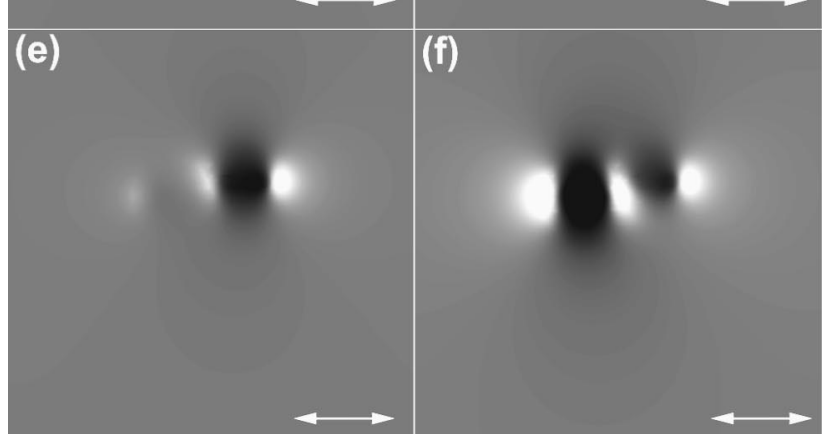

FIG. 3. Simulated images reconstructed from the AFM data of Fig. 2 assuming $R_{\mathrm{tip}}=10 \mathrm{~nm}$, index $n=1.6$, and the external source polarization along the direction indicated by the arrow. (a),(b) polarizations dependence; (c) $R_{\text {tip }}=15 \mathrm{~nm}$; (d) $R_{\text {tip }}=30 \mathrm{~nm}$; (e) Left bead $n=1.1$; and (f) Left bead $n=4.0$. White indicates highest intensity.

Downioaded 03 Apr 2006 to 131215.2

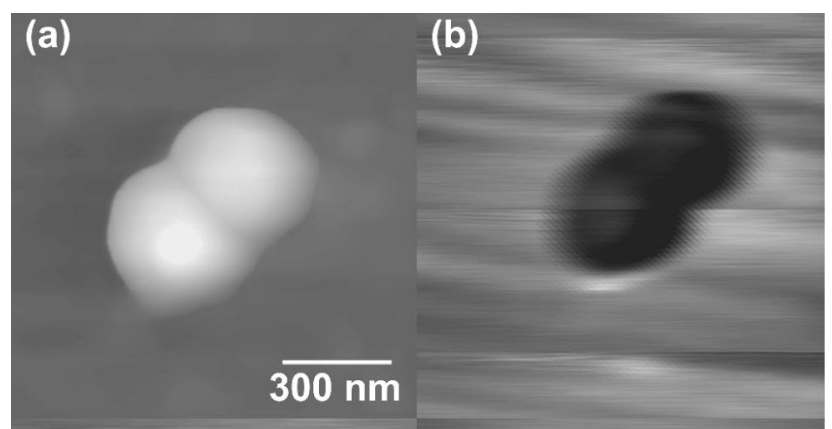

(c)

(d)

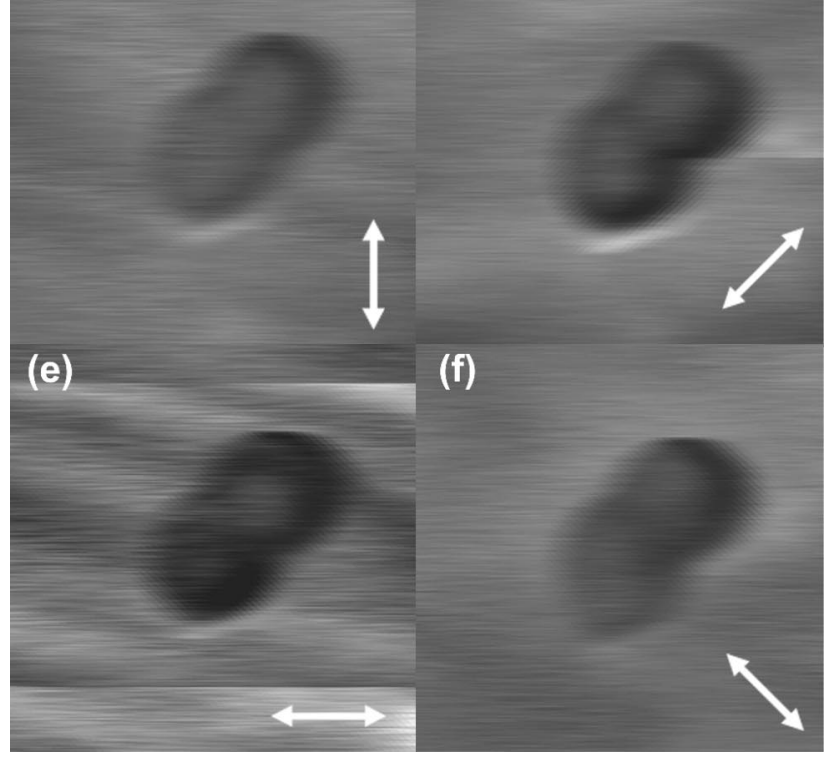

FIG. 4. Experimental data on $200 \mathrm{~nm}$ polystyrene beads. (a) AFM image; (b) SAM image with external laser composed of mixed polarizations; and (c)-(f) SAM images with the external laser linearly polarized in the direction indicated by the white arrow.

field of the sample, accurate to first order in the external field $\mathbf{E}_{\text {laser }} \cos \omega t$, and taking into account the finite dimensions of the dipole, is

$$
\begin{aligned}
\mathbf{E}_{s}= & \frac{\alpha_{s}\left|\mathbf{E}_{\text {laser }}\right|}{2 R_{s}^{3}}\left(\left[\frac{(R-\cos \theta) \cos \omega t_{-}}{d_{+}^{3 / 2}}\right.\right. \\
& \left.-\frac{(R+\cos \theta) \cos \omega t_{+}}{d_{-}^{3 / 2}}-\frac{\omega R_{s} \sin \omega t_{-}}{c d_{+}}-\frac{\omega R_{s} \sin \omega t_{+}}{c d_{-}}\right] \hat{e}_{r} \\
& +\sin \theta\left[\frac{\cos \omega t_{-}}{d_{+}^{3 / 2}}+\frac{\cos \omega t_{+}}{d_{-}^{3 / 2}}-\frac{\omega R_{s} \sin \omega t_{-}}{c d_{+}}\right. \\
& \left.\left.-\frac{\omega R_{s} \sin \omega t_{+}}{c d_{-}}\right] \hat{e}_{\theta}\right),
\end{aligned}
$$

where $r$ is the distance between the center of the sample and the observation point, $\theta$ is the angle between the sample's dipole moment and the vector locating the observation point, $\omega$ is the angular frequency of the external laser, $t$ is time, $d_{ \pm}=1+R^{2} \pm 2 R \cos \theta, t_{ \pm}=t-R_{s} d_{ \pm}^{1 / 2} / c$, and $R=r / R_{s}$. This near field, $E_{s}$, changes the induced dipole moment of the AFM tip and effectively modulates the scattered intensity measured at the detector as the AFM tip scans across the sample. In this first-order treatment the effect of the tip on the sample field is ignored. The near field of the sample dipole will add destructively with the external field inducing
to AlP license or copyright, see http://apl.aip.org/apl/copyright.jsp 
the tip dipole when the tip is directly above the sample. The fields will add constructively when the tip is on either side of the sample along the dipole axis, although this effect is more localized and, therefore, presumably more difficult to observe than the effects due to destructive interference.

We have imaged polystyrene nanospheres of diameters ranging from 30 to $200 \mathrm{~nm}$. The AFM image in Fig. 2 is of two adjacent $50 \mathrm{~nm}$ diam polystyrene beads on glass. Also shown is apertureless data taken with the illuminating laser randomly polarized. In Fig. 3 we present the results of the simulations which show the important features of the dipole model. The AFM image is used as a topographical template for the simulations since the dipole field influencing the tip will be a function of the center-to-center separation between tip and sample. To the topography we add the locations and other pertinent information about the sample dipoles induced by the external laser polarization. With this information we can determine the scattered intensity at any point to construct the final image. Unless otherwise stated, all simulations use the parameters $R_{\text {tip }}=10 \mathrm{~nm}, n_{\text {sample }}=1.6, \lambda_{\text {laser }}=632.8 \mathrm{~nm}$. The scales in the simulations are normalized to unity for the level of background scattering off of the AFM tip in the absence of modulation due to the sample's electric field. All are linear plots of the normalized intensity with full scale from 0.70 (black) to 1.8 (white). Values outside this intensity range are clipped to the minimum or maximum value accordingly. Figures 3(a) and 3(b) show how the near field of the dipole orients itself with the polarization of the external laser source. Figures 3(a) and 3(c) suggest that an enhancement in resolution could be obtained with the proper orientation of the source polarization. Our initial experiments have shown a definite dependence on the polarization of the external laser source. Figures 3(c) and 3(d) show how edge sensitivity decreases with increasing tip size. Although a larger tip radius means a larger scattering cross section, and hence, more signal, the change in signal intensity with respect to the background scattering is reduced with a larger tip. Figures 3(e) and 3(f) show how the image intensity changes for features with different indices of refraction. These images show clearly that the dipole model can image differences in optical properties.

Experimental images with polarization dependent features are shown in Fig. 4. The samples being imaged are two $200 \mathrm{~nm}$ polystyrene spheres adsorbed on a glass substrate. The optical images in Figs. 4(b)-4(f) show the lower intensities above the sample due to destructive interference between the near field of the sample and the external laser shown in the simulations. As the direction of linear polarization of the external laser is changed, stark differences in contrast are evident. For example, the bright area in the image in Fig. 4(d) is no longer apparent with the orthogonal polarization shown in Fig. 4(f), resembling the polarization dependence shown in the simulations in Figs. 3(a) and 3(b).

In conclusion, we have used a simple dipole-dipole scattering model to demonstrate that our apertureless technique can, in theory, image information about a sample that is indeed optical in nature. Our model contains no fitting parameters; all physical constants used are well known or easily measured. Initial experiments show a strong polarization dependence in the images, as well as destructive interference lowering the signal intensity when the tip is above the sample. We conclude that many features of the dipole coupling model are in qualitative agreement with experimental data.

This work was supported under Office of Naval Research Contract No. N00014-98-1-0567 and by a grant from Hughes Research Laboratories, Inc., LLC.

${ }^{1}$ H. K. Wickramasinghe and C. C. Williams, Apertureless Near Field Optical Microscope, U.S. Patent No. 4,947,034 (April 28, 1989).

${ }^{2}$ F. Zenhausern, M. P. O’Boyle, and H. K. Wickramasinghe, Appl. Phys. Lett. 65, 1623 (1994).

${ }^{3}$ B. Hecht, H. Bielefeldt, Y. Inouye, L. Novotny, and D. W. Pohl, J. Appl. Phys. 81, 2492 (1997).

${ }^{4}$ P. M. Bridger and T. C. McGill, Opt. Lett. 24, 1005 (1999).

${ }^{5}$ J. D. Jackson, Classical Electrodynamics, 2nd ed. (Wiley, New York, 1975). 